\title{
DESIGN OF A HARMONIC GENERATION FEL EXPERIMENT AT BNL"
}

I. Ben-Zvi, A. Friedman, C.M. Hung, G. Ingold, S. Krinsky, K.M. Yang, L.H. Yu NSLS, Brookhaven National Laboratory, Upton NY 11973

I. Lehrman, D. Weissenburger

Grumman Corp. Independence Way, Princeton NJ 08540

$$
\text { An } 092
$$

\begin{abstract}
We present the design parameters of a harmonic generation FEL experiment to be carried out at the Accelerator Test Facility (ATF) at BNL. This experiment will je carried out as a proof-of-principle for the proposed UV-FEL User's Facility at BNL. In the experiment we plan to triple the frequency of $\mathrm{a} \mathrm{CO}_{2}$ seed laser by utilizing two superconducting wigglers and a dispersive section. The first wiggler will be used in conjunction with the $\mathrm{CO}_{2}$ seed laser to generate a ponderomotive force shat will bunch the electron beam. The bunching will then be enhanced by the dispersion section. The second wiggler, tuned to the third harmonic of the seed laser will follow. In the beginning 0 ? the second wiggler the bunched beam will produce super-radiant emission (characterized by a quadratic growth of the radiated power), then the radiation will be amplified exponentially. The last part of the wiggler will be tapered. We plan to study the evolution of the various radiation growth mechanisms as well as the coherence of the tripled and exponentially amplified radiation.
\end{abstract}

"This work is performed uncier the auspices of the U.S. Department of Energy under contract number DE-AC02-76-Cio0016 and the Grumman Corporation. 


\section{INTRODUCTION}

The seeded single pass FEL has many advantages over other FEL concepts. The output bandwidth is controlled by the input seed, limited only by the pulse length, and a bandwidth of $10^{-4}$ is possible. Similarly, the frequency stability is also controlled by the seed; hence the electron beam energy stability influences only the output intensity fluctuations, and the requirement on the energy stability is largely relaxed. Another obvious advantage is that the mirror loss and damage problems of FEL oscillators are eliminated. In addition, there is no need for a long train of micropulses. The electron beam can consist of single micropulses with the high repetition rate available from a superconducting linac. Thus, it is possible to achieve very good energy stability and high average power.

There are powerful, high repetition-rate tunable lasers operating in the IR and visible frequency bands which may be harmonic-multiplied into the VUV and used as seed lasers for the FEL amplifier. The interest in harmonic generation in FELs stems from the limitations of conventional laser harmonic generation techniques, such as low conversion efficiency, susceptibility to damage and limited tunability.

The generation of harmonics by bunching an electron beam in a wiggler, using a seed laser, is well known and verified experimentally [1]. The use of prebunching in FELs was studied analytically [2] and more recently, in a 3-D numerical simulation [3]. The extension of this technique to the exponential growth regime including wiggler tapering has been proposed and studied in detail as the basis for the UV-FEL User's Facility at BNL [4] [5] [6]. An order of magnitude improvement in the performance of this system can be obtained by further modifications of the technique [7]. However, the complete process of generating the harmonics by prebunching in the fundamental and amplification in a wiggler tuned to the harmonic has not been demonstrated experimentally as yet.

The generation of harmonics and subsequent exponential growth poses many interesting questions. At present, we are addressing some of these issues by theoretical research. However, it is our intention to pursue these questions experimentally.

In the proposed harmonic generation experiment, we will demonstrate the bunching of a $30 \mathrm{MeV}$ electron beam by a $\mathrm{CO}_{2}$ laser of nearly $1 \mathrm{MW}$ input power. We will study the 
super-radiant growth of the third harmonic at a wavelength of $3.47 \mu \mathrm{m}$, the exponential growth regime, and finally a tapered wiggler FEL amplifier section. We would like to verify our theoretical models and to answer important questions such as the effect of electron beam parameters on the coherence of the FEL, the effect of wiggler and alignment errors, and the higher harmonic contents of the FEL output as a function of the level of saturation.

The harmonic generation experiment is proposed for the BNL Accelerator Test Facility (ATF) [8]. We have selected the parameters of the harmonic generation experiment to match the electron beam parameters which have already been demonstrated experimentally at the ATF. These include a normalized rms emittance of $4 \pi \mathrm{mm}$ mrad at a peak current of 130 amperes. The energy of $30 \mathrm{MeV}$ is well within the ATF design of $50 \mathrm{MeV} . \mathrm{A} \mathrm{CO}_{2}$ oscillator with a pulse length of nearly 100 ns and a power of $1 \mathrm{MW}$ is available. In addition, the ATF has a solid-state optical chopper on the $\mathrm{CO}_{2}$ laser system which is synchronized to the electron beam. This chopper is capable of slicing a 10-100 picosecond long pulses. If necessary, that pulse can be amplified by a wide-band $\mathrm{CO}_{2}$ amplifier.

\section{PARAMETERS OF THE HARMONIC GENERATION EXPERIMENT}

The design and theory of the harmonic generation FEL has been described extensively elsewhere [5]. Therefore we will confine this presentation to a brief description of the parameters of the experiment and the expected performance of the system.

Table 1 provides all the relevant parameters of the experiment: seed laser, electron beam, wiggler, and expected FEL amplifier performance. The FEL parameters have been optimized using a procedure, described in reference [5] which relies on an semi-analytic model of a 3-D exponential regime FEL [9]. The optimized parameters are the seed laser input power and Rayleigh range, the wiggler wavelength, the electron beam energy, the strength of the dispersive section, the taper start position, and the rate of tapering.

Following the optimization of the FEL parameters, the complete simulation of the harmonic generating FEL has been done using a modified version of the computer code TDA $[10]$.

A schematic diagram of the harmonic generation experiment is shown in Figure 1. 
We will use a $\mathrm{CO}_{2}$ seed laser at a wavelength of $10.4 \mu \mathrm{m}$. The seed-laser pulse-length will be initially $100 \mathrm{~ns}$ at an input power of $0.9 \mathrm{MW}$. The electron-beam pulse-length at the ATF is from 6 to 10 ps FWHM.

Our system utilizes two wiggler magnets separated by a dispersion section. The first wiggler, which is $30 \mathrm{~cm}$ long, is used to energy modulate the electron beam. The period of this wiggler is $2.8 \mathrm{~cm}$ and the peak axial magnetic field is $0.71 \mathrm{~T}$, making the $30 \mathrm{MeV}$ electron beam resonant with the $10.4 \mu \mathrm{m}$ seed radiation. This is followed by a $10 \mathrm{~cm}$ long dispersion section with a magnetic field of $0.9 \mathrm{~T}$, in which this energy modulation is converted into a spatial bunching with a strong third harmonic component at $3.47 \mu \mathrm{m}$.

When the coherently bunched beam enters the $1.5 \mathrm{~m}$ long radiator wiggler magnet (resonant at $3.47 \mu \mathrm{m}$ ), there is a rapid coherent generation of $3.47 \mu \mathrm{m}$ radiation within the first few wiggler periods. The radiation has a characteristic quadratic dependence on the distance traverser: in the wiggler. Then there is a transition to exponential growth which continues until $0.7 \mathrm{~m}$ into the wiggler, where the $3.47 \mu \mathrm{m}$ radiation approaches saturation. Starting at $0.3 \mathrm{~m}$ from the beginning of the radiator wiggler, a $1.2 \mathrm{~m}$ long tapered section is used to optimize the power extracted from the electron beam.

The three distinct FEL processes occuringin the second wiggler (the quadratic superradiant growth, the exponential growth, and the quadratic growth towards the end of the tapered section) are shown clearly when the radiation power plotted against the wiggler length in Fig. 2.

The optimization procedure shows that a quadratic tapering which starts from $0.3 \mathrm{~m}$ maximizes the output power even though the radiation is still well in the exponential growth regime. Thus, we note that there is exponential growth in the tapered section of the wiggler, since the taper is very gradual and the Pierce parameter is large, $\rho_{0}=0.0074$.

We have looked into the FEL performance sensitivity to electron beam parameters, such as the emittance and energy spread. Doubling the emittance, from $4 \times 10^{-6}$ to $8 \times$ $10^{--6} \pi \mathrm{m}$ rad changes the power e-folding length of the second wiggler from $0.143 \mathrm{~m}$ to $0.174 \mathrm{~m}$. Similarly, changing the FWHM energy spread from $0.1 \%$ to $0.2 \%$ changes the e-folding length from $0.143 \mathrm{~m}$ to $0.147 \mathrm{~m}$. We notice that neither parameter is very critical, particularly the energy spread. One can even increase the emittance to $6 \times 10^{-6} \pi \mathrm{m}$ rad 
and at the same time the energy spread to $0.2 \%$, and the e-folding length changes to 0.164 m. Thus, we conclude that the experiment is designed rather conservatively.

\section{DESIGN OF THE HARMONIC GENERATION EXPERIMENT}

Since one of our goals is ":o study the FEL interaction in the second wiggler, we would like observe conveniently the evolution of the radiation in the wiggler. We plan to place the optical diagnostics at the output end of the wiggler, along the beam axis. The electron beam will be bent out of the way. The fundamental radiation, although much weaker in peak power, extends over a much longer time than the signal. We expect that a significant amount will not be diffracted out of the optical diagnostic's acceptance thus there is a potential for a poor signal to noise problem.

This background can be reduced in two ways. The simple approach is to place a magnesium fluoride high pass filter which will cut out the $10.4 \mu \mathrm{m}$ fundamental but will not attenuate significantly the $3.47 \mu \mathrm{m}$ harmonic. Additional reduction of the $10.4 \mu \mathrm{m}$ background by many orders of magnitude can be obtained by chopping the long $\mathrm{CO}_{2}$ oscillator pulse using germanium transmission and reflection switches. These germanium plates are switched by the $1.064 \mu \mathrm{m}$ of the Nd:YAG laser which drives the photocathode rf gun. Thus, the resultant sliced $\mathrm{CO}_{2}$ pulse is synchronized to the electron micropulse.

We plan to turn on the wiggler one section at a time and observe the growth of the FEL power. For this purpose, we will use an electromagnet wiggler constructed of many short sections which may be powered independently. The technology of a wiggler suitable for this purpose has been developed at the National Synchrotron Light Source at BNI [11].

We use a ferromagnetic yoke machined out of a solid block of low carbon steel. A superconducting $\mathrm{NbTi}$ coil is wound continuously along the yoke, with the winding direction alternating every half period.

The magnetic field of this undulator is very uniform even for operation above saturation. We have demonstrated that the rms random error for the peak field can be less than $0.30 \%$ for the highest possible current densities. To obtain such a performance no post-machining trimming or shimming whatsoever is applied besides the operation of correction trim coils 
on either end of the magnets. The field accuracy depends mainly on the tight machining tolerances (10 microns) which can be met by precision Wire Electric Discharge Machining. This error is small enough so that the costly error reduction procedure applied in the hybrid wiggler technology is eliminated.

The wiggler for the harmonic generation experiment has been evaluated by 2-D POISSON model calculations. The wiggler design is similar to that of the FEL oscillator at the ATF [11]. That particular undulator has a period of $8.80 \mathrm{~mm}$ and a gap of $4.40 \mathrm{~mm}$. These dimensions have been scaled accordingly to a $16.0 \mathrm{~mm}$ period and a $6.0 \mathrm{~mm}$ gan. Using the same wire, wound in $34(=9+8+9+8)$ turns per half-pole in 4 layers, we can expect a maximum current of 170 amperes. This current is $20 \%$ lower than the quench current estimated from the load curve analysis. Under these conditions, a field of $0.88 \mathrm{~T}$ on axis is predicted.

At this axial field the iron is saturated. For the design of the harmonic generation experiment, we apply a scale-down factor of 0.80 , based on a comparison of measurements and POISSON calculations for the ATF FEL undulator.

These estimates are based on the assumption of a flat pole wiggler. As can be seen in Table 1, the betatron wavelength of this wiggler is nearly equal to the total length of the wiggler. Therefore we plan on using plane focussing wiggler with equal focussing in both transverse dimensions [12]. The 3-D magnetic design of the wiggler has not been done at this point, but the 2-D calculation is far enough from the maximum current density possible to give us confidence that the necessary $3-D$ field profile can be achieved.

\section{References}

[1] R. Prazeres, P. Guyot-Sionnest, J.M. Ortega, D. Jaroszynski, M. Billardon, M.E. Couprie, M. Velghe and Y. Petroff, Coherent harmonic generation in VUV with the optical klystron on the storage ring Super-ACO, Nucl. Instr. and Meth. A304, 72 (1991).

[2] I. Boscolo and V. Stagno, A study of a transverse optical klystron experiment in Adone (TOKA), Nucl. Instr. and Meth. 198, 483 (1982). 
[3] Generation of XUV Light by Resonant Tripling in a Two Wiggler FEL Amplifier. R. Bonifacio, L. de Salvo Souza, P. Pierini and E.T. Scharlemann, Nucl. Instr. and Meth. A296, 787 (1990).

[4] I. Ben-Zvi, L.F. Di Mauro, S. Krinsky, M. G. White and L.H. Yu, Proposed UVFEL User Facility at BNL, Proceedings of the 1990 International Free-Electron Laser Conference, Paris September 1990.

[5] L.H. Yu, Generation of Intense UV Radiation by Subharmonically seeded Single Pass FEL, Accepted and to be published in Phys. Rev. A. BNL 45970, 1991.

[6] I. Ben-Zvi, L. F. Di Mauro, S. Krinsky, M. G. White L. H. Yu, K. Batchelor, A. Friedman, A.S. Fisher, H. Halama, G. Ingold, E. D. Johnson, S. Kramer, J.T. Rogers, L. Solomon, J. Wachtel and X. Zhang. Proposed UV-FEL User Facility at BNL Proceedings of the 1991 International FEL Conference, Santa Fe, NM.

[7] I. Ben-Zvi, K.M. Yang and L.H. Yu, The 'Fresh Bunch' Technique in FELs Proceedings of the 1991 International FEL Conference, Santa Fe, NM.

[8] I. Ben-Zvi, The BNL Accelerator Test Facility and Experimental Program, Proceedings of the 1991 Particle Accelerator Conference, San Francisco CA.

[9] L.H. Yu, S. Krinsky and R.L. Gluckstern, Calculation of universal scaling function for FEL gain, Phys. Rev. Lett. 64 No. 25, 3011, (1990).

[10] T.M. Tran and J.S. Wurtele, LRP 354/88 Echole Polytechnique Federale de Lausanne - Suisse, 1988.

[11] I. Ben-Zvi, R. Fernow, J. Gallardo, G. Ingold, W. Sampson and M. Woodle, Performance of a Superferric, High Field Subcentimeter Undulator Proceedings of the 1991 International FEL Conference, Santa Fe, NM.

[12] E.T. Scharlemann, Wiggle plane focussing in linear wigglers, J. Appl. Phys. 58,2154 (1985).

\section{DISCLAIMER}

This report was prepared as an account of work sponsored by an agency of the United States Government. Neither the United States Government nor any agency thereof, nor any of their employees, makes any warranty, express or implied, or assumes any legal liability or responsibility for the accuracy, completeness, or usefulness of any information, apparatus, product, or process disclosed, or represents that its use would not infringe privately owned rights. Reference herein to any specific commercial product, process, or service by trace name, trademark, manufacturer, or otherwise does not necessarily constitute or imply its endorsement, recommendation, or favoring by the United States Government or any agency thereof. The views and opinions of authors expressed herein do not necessarily state or reflect those of the United States Government or any agency thercof. 
Table 1. System parameters

\begin{tabular}{|l|c|l|}
\hline Electron beam parameters & & \\
\hline Energy, $\gamma$ & 60.594 & \\
Current, $\mathrm{I}$ & 110 & amperes \\
Micropulse length & 10 & $\mathrm{ps}$ \\
Emittance (normalized, rms) & $4 \times 10^{-} 6$ & $\pi \mathrm{m}$ rad \\
Beam edge radius (matched beam) & 0.34 & $\mathrm{~mm}$ \\
Local energy spread $\sigma_{\gamma} / \gamma$ & 0.043 & $\%$ \\
\hline Seed laser parameters & & \\
\hline Input power $\mathrm{P}_{\text {in }}$ & 0.9 & $\mathrm{MW}$ \\
Pulse length, initial & 100 & $\mathrm{~ns}$ \\
\hline Wiggler parameters & & \\
\hline Subharmonic wiggler: & & \\
Length $\mathrm{L}_{S W}$ & 0.3 & $\mathrm{~m}$ \\
Period $\lambda_{S W}$ & 2.8 & $\mathrm{~cm}$ \\
Peak axial magnetic field $\mathrm{B}_{S W}$ & 0.71 & $\mathrm{~T}$ \\
Dispersive section: & & \\
Length $\mathrm{L}_{D}$ & 10 & $\mathrm{~cm}$ \\
Magnetic field $\mathrm{B}_{D}$ & 0.9 & $\mathrm{~T}$ \\
Main wiggler: & & \\
Period $\lambda_{W}$ & 1.6 & $\mathrm{~cm}$ \\
Exponential section length $\mathrm{L}_{E}$ & 0.3 & $\mathrm{~m}$ \\
Exponential section magnetic field $\mathrm{B}_{W}$ & 0.729 & $\mathrm{~T}$ \\
Magnetic gap $\mathrm{g}$ & 6 & $\mathrm{~mm}$ \\
Betatron wavelength $\lambda_{\beta}$ & 1.8 & $\mathrm{~m}$ \\
Tapered section length $\mathrm{L}_{T}$ & 1.2 & $\mathrm{~m}$ \\
Field taper & 10 & $\%$ \\
\hline FEL parameters & & \\
\hline Bessel factor $J_{0}-J_{1}$ & 0.818 & \\
Exponential section power e-folding length & 0.143 & $\mathrm{~m}$ \\
Pierce parameter $\rho_{0}$ & 0.0074 & \\
Output power & 52 & $\mathrm{MW}$ \\
Output energy for a 10 pS FWHM pulse & $\sim 0.2$ & $\mathrm{~mJ}$ \\
\hline
\end{tabular}




\section{FIGURE CAPTIONS}

1. Schematic diagram of the harmonic generation experiment.

2. Logarithm of the radiation power along position in the wiggler of the $3.47 \mathrm{mum}$ section. 


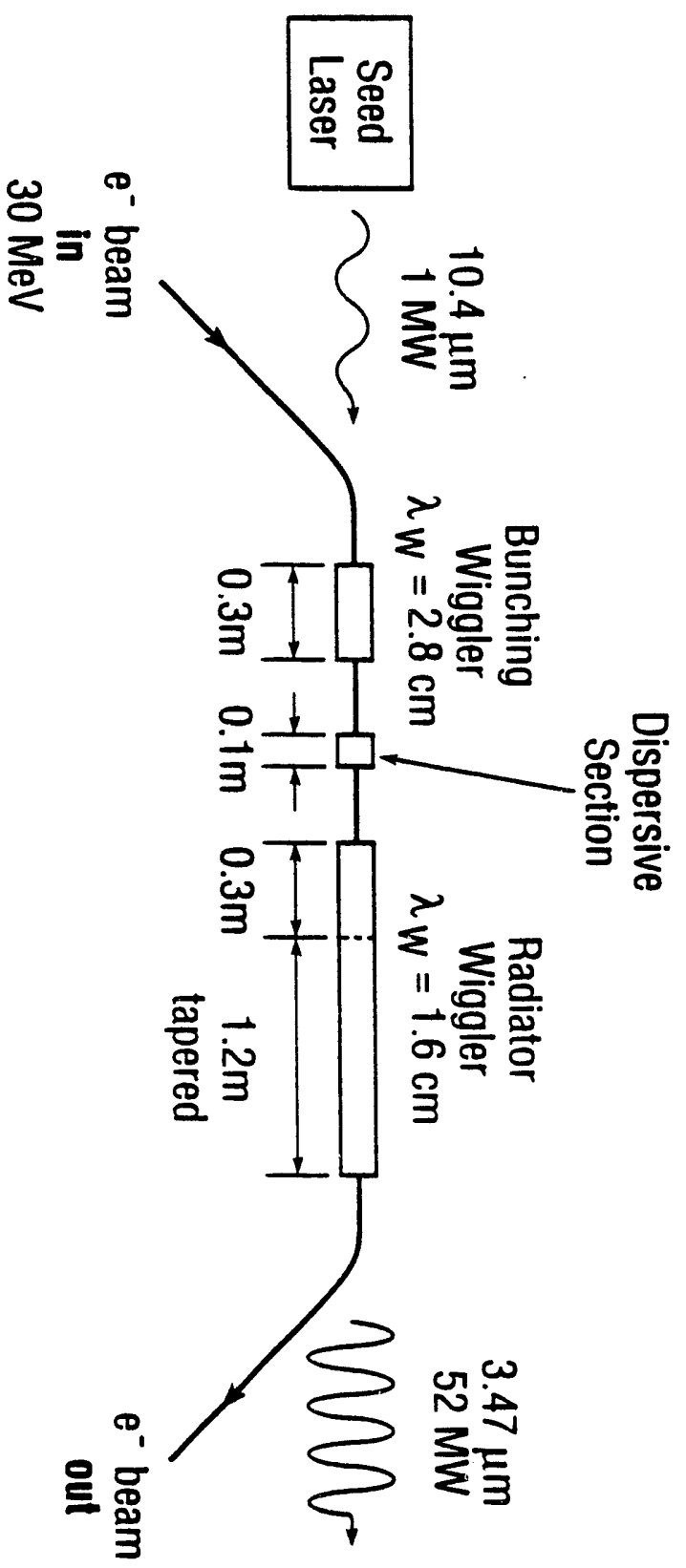




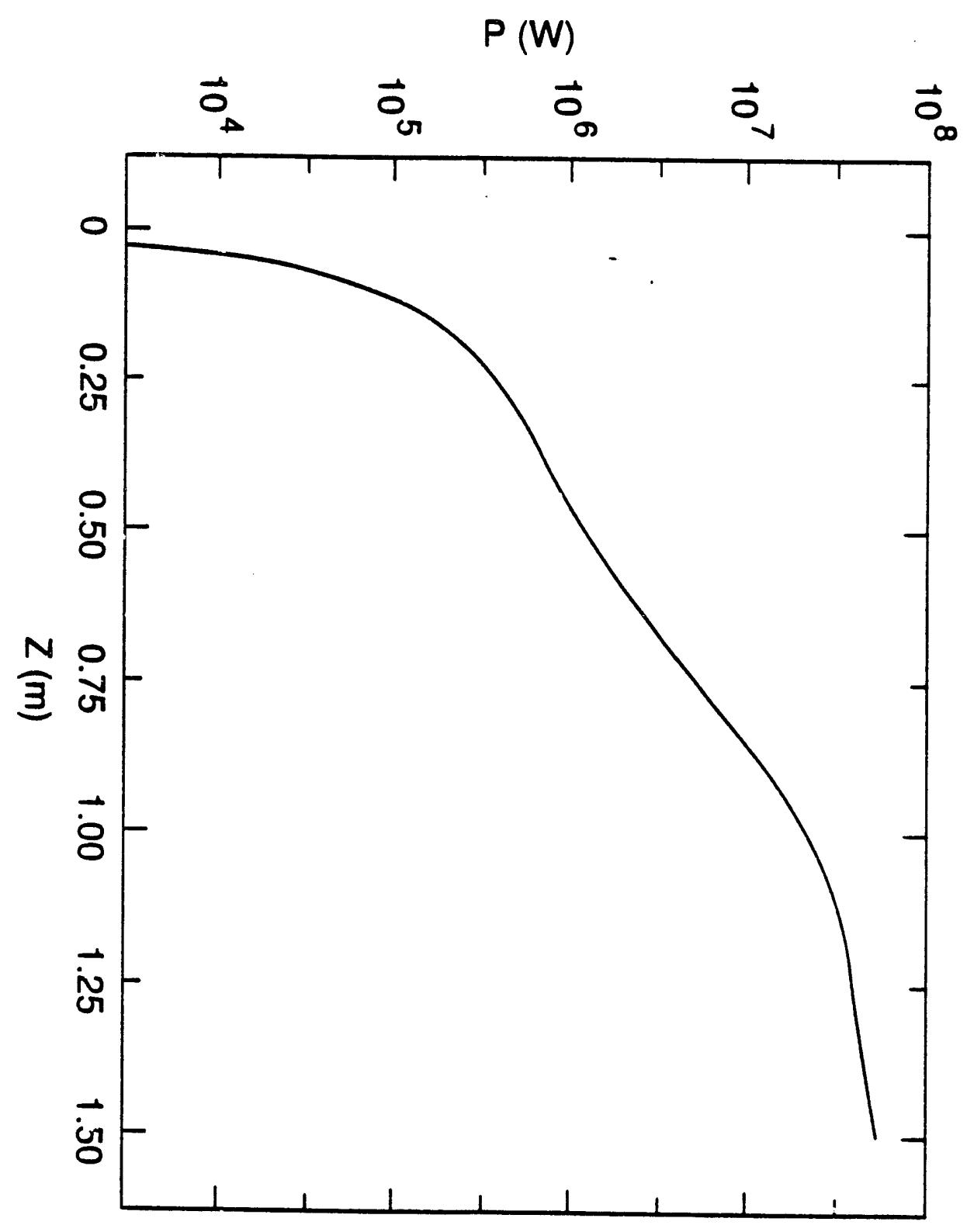

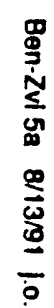



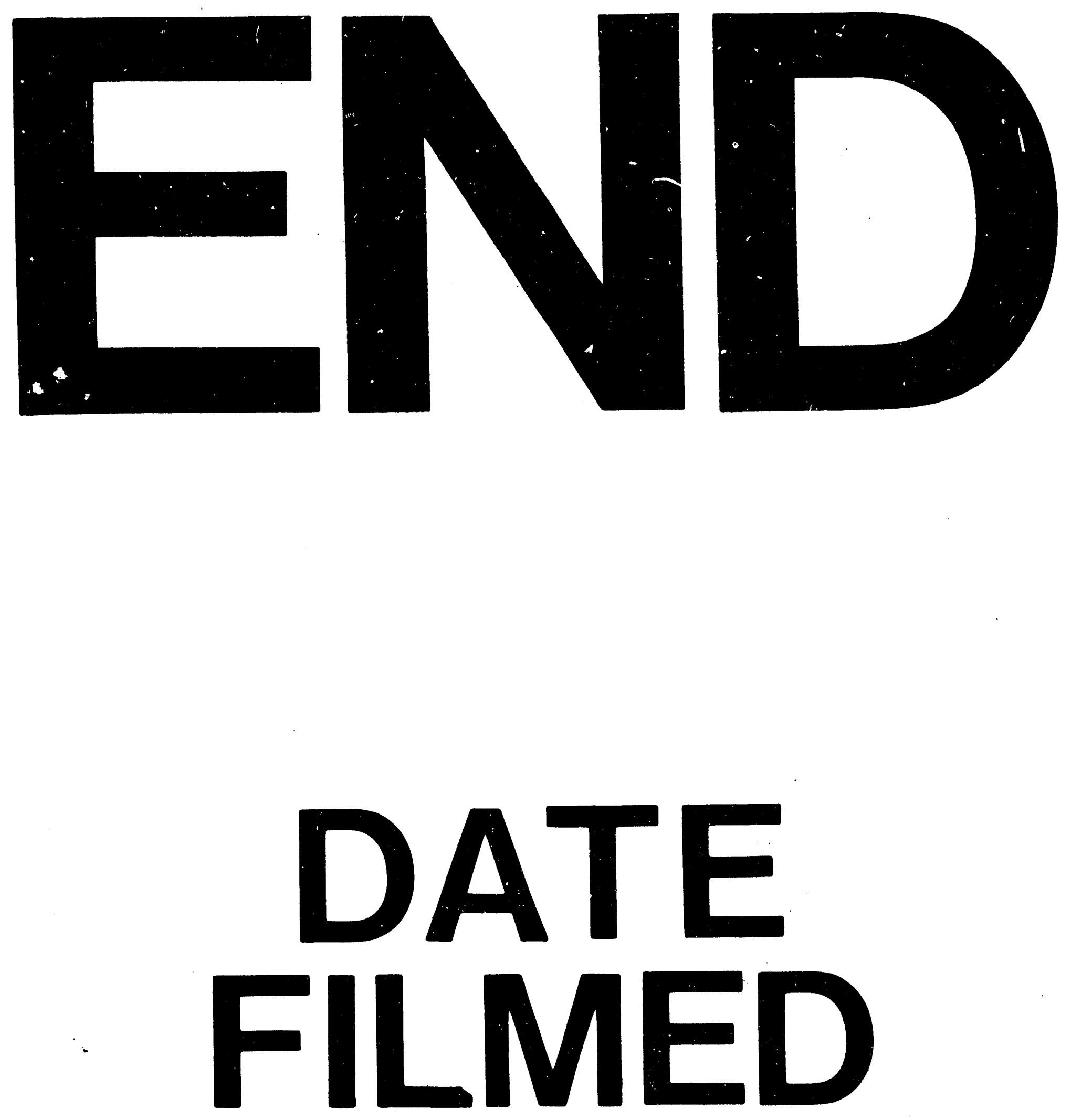

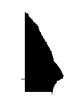

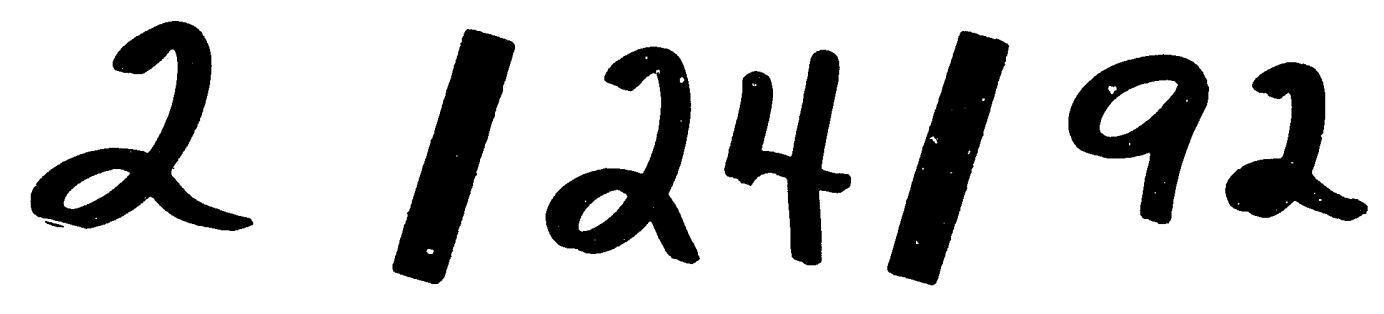

